\title{
Zur Kenntnis der Fließvorgänge bei hochpolymeren Verbindungen
}

\author{
Von Walter Scheele \\ Aus der Kolloid-Abteilung des Instituts für Physikalische Chemie \\ der Technischen Hochschule Hannover \\ (Z. Naturforschg. 4 a, 433-439 [1949]; eingegangen am 10. August 1948)
}

\begin{abstract}
In Fortführung und Erweiterung früherer Untersuchungen über die Rheometrie organischer Gläser wird gezeigt, daß die Fließkurven von Polyvinylacetaten Potenzfunktionen sind, deren Beschreibung mit nur einem Exponenten $n$ gelingt. Seine Temperaturabhängigkeit wird diskutiert. Die doppeltlogarithmischen Fließkurven ergeben eine weitere Konstante $K$, die die Fließgeschwindigkeit für die Einheit der Schubspannung darstellt. Mit Hilfe von $n$ und $K$ kann die bereits in früheren Abhandlungen definierte Verformungsleistung für verschiedene Fließgeschwindigkeiten berechnet werden. Die spezifische Verformungsleistung ist die Verformungsleistung bei der Einheit der Fließgeschwindigkeit. Die Temperaturfunktion der Verformungsleistung wird diskutiert. Es besteht ein funktioneller Zusammenhang zwischen ihrer Richtungskonstanten und dem Polymerisationsgrad innerhalb einer polymerhomologen Reihe, so daß sich eine rheologische Regel für lösungsmittelfreie Kettenpolymerisate ergibt, die Fließvermögen und Polymerisationsgrad miteinander verknüpft.
\end{abstract}

I.

$\mathrm{B}^{\mathrm{o}}$ ei praktisch sämtlichen Methoden der Verarbeitung von Polymerisaten und Polykondensaten spielen die Fließvorgänge eine überragende Rolle. Trotz ihrer Bedeutung und obwohl zahlreiche fließkundliche Experimentaluntersuchungen vorliegen ${ }^{1}$, ist über die Zusammenhänge zwischen rheologischem Verhalten eines in Frage stehenden Materials und seiner chemischen Konstitution sowie seinem inneren Aufbau eigentlich recht wenig bekannt.

Seit einigen Jahren beschäftigen wir uns mit rheologischen Untersuchungen an Polymerisaten ${ }^{2}$. Ihr hauptsächliches Ziel ist die Aufdeckung von Zusammenhängen zwischen dem Fließvermögen und vornehmlich stofflich bedingten Eigenschaften der Polymerisate. Die Arbeiten konnten inzwischen fortgesetzt und erweitert werden. Die Mitteilung ihrer Ergebnisse bildet den Gegenstand dieser Abhandlung.

II.

Wenn man auf ein isotropes Polymerisat oder Polykondensat eine Kraft (Schubspannung) einwirken läßt, so tritt eine Verformung ein, deren

1 S c ott B lair, Einführung in die Fließkunde, Dresden u. Leipzig 1940; T. A l f r e y, Mechanical Behavior of High Polymers, New York 1948.

2 W. Sch e e le u. Mitarbb., Kolloid-Z. 103, 1 [1943]; 105, 210 [1943]; 108, 44 [1944].
Geschwindigkeit mit der Temperatur und der Schubspannung zunimmt. Sie kann völlig elastisch oder plastisch und schließlich auch von elastischplastischem Charakter sein. Ob das eine oder andere beobachtet wird, hängt von der Versuchstemperatur, der Einwirkungsdauer der Kraft, von Konstitution, Größe und Gestalt der Moleküle des betreffenden Körpers sowie von dessen übermolekularer Struktur ab. Bei den beschriebenen Verformungserscheinungen spielen Fließvorgänge eine Rolle.

Hauptsächlich 4 Typen von Fließkurven werden bei der Verformung plastischer Massen beobachtet $^{3}$. Sie sind in der Abb. 1 zusammengestellt (Ordinate: Fließgeschwindigkeit $v$; Abszisse: Schubspannung $\tau$ ). In den Fällen a) und b) ist $v$ eine lineare Funktion der Schubspannung (Newtonsche Systeme mit und ohne Fließgrenze $F$ ); bei c) und d) besteht überproportionale Abhängigkeit zwischen $v$ und $\tau$ (nicht-Newtonsche Körper mit und ohne Fließgrenze $F$ ).

$\mathrm{Ob}$ ein gegebenes Material eine Fließgrenze hat, kann nicht immer mit völliger Sicherheit entschieden werden. Falls die Fließkurve eine Gerade ist,

3 R. H o uw in k, Elastizität, Plastizität und Struktur der Materie, Dresden u. Leipzíg 1934; Physikalische Eigenschaften und Feinbau von Natur- und Kunstharzen, Leipzig 1934; Kunststoff-Techn. 11, 228 [1941]; F. H. Müller, Physik und Kolloidstruktur, in R. Houw in k, Chemie und Technologie der Kunststoffe, Leipzig 1942, Bd. I, S. $198 \mathrm{ff}$. 
kann man eine Extrapolation auf die Fließgeschwindigkeit Null vornehmen. Man unterstellt dabei natürlich, daß die Kurve auch im Bereich kleinster Fließgeschwindigkeiten ihre Richtungskonstante behält. Es empfiehlt sich aber immer, eine auf diese Weise ermittelte Fließgrenze als ,praktische Fließgrenze“ zu bezeichnen und ihr in wissenschaftlicher Hinsicht keine zu große Bedeutung beizumessen. Das gilt naturgemäß ganz besonders für nicht-Newtonsche Systeme, bei denen die Bestimmung einer Fließgrenze noch größere Schwierigkeiten bereitet. Bedenkt man, daß es in einer Flüssigkeit mit fixierter Struktur ${ }^{4}$,
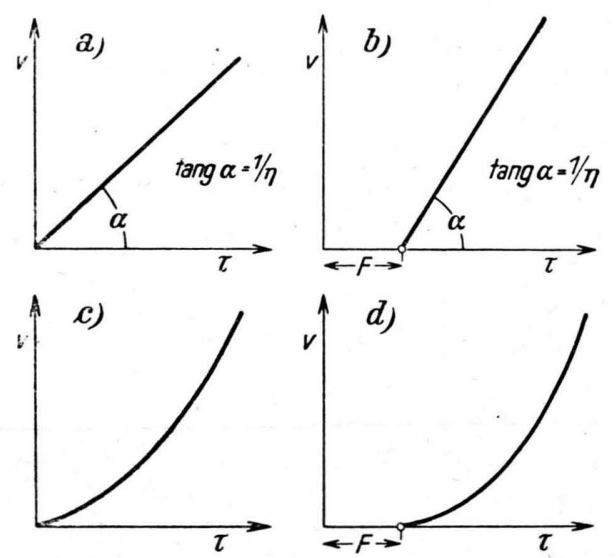

Abb. 1. Verschiedene Typen von Fließkurven. a) und b) Newtonsche Systeme mit bzw. ohne Fließgrenze $F$;

c) und d) nicht-Newtonsche Körper mit bzw. ohne Fließgrenze $F$.

also in einem isotropen Körper mit hoher Zähigkeit, sehr unterschiedlicher Energiebeträge bedarf, um die Strukturelemente aus ihren Gleichgewichtslagen $\mathrm{zu}$ entfernen, so gelangt man $\mathrm{zu}$ der Vorstellung, daß es nur eine Frage der Beobachtungszeit ist, ob eine Fließgrenze angenommen werden muß oder nicht. Eine bei einigermaßen rasch durchgeführter Verformung auftretende Fließgrenze sollte danach bei lange andauernder Einwirkung der deformierenden Kraft (auch der kleinsten Kraft) zum Verschwinden gebracht werden können.

Während man Newtonsche Systeme durch die Viskosität bzw. deren reziproken Wert, die Fluidität $\varphi$, charakterisieren kann, Größen, die für angenommene Temperaturen als Materialkonstanten angesehen werden können, liegen die

* K. Ueberreiter, Z. angew. Chem. 53, 247, 259 [1940]; Kunststoffe 30, 170 [1940]; Z. physik. Chem., Abt. B 45, 361 [1940]; 46, 157 [1940]; 48, 197 [1941].
Formelübersicht.

$$
\begin{aligned}
& v=\frac{1}{\eta} \tau \\
& v=\frac{1}{\eta}\left(\tau-F^{\prime}\right) \\
& v=K \tau^{n}
\end{aligned}
$$

$\log K=\log v-n \log \imath$

$$
v=K(\tau-F)^{n}
$$

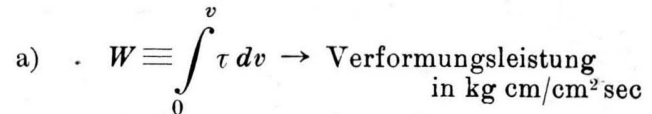

b) $1 / W \equiv \varphi_{W} \equiv 1 / \int \tau d v \rightarrow$ Verformbarkeit $W \equiv \int_{0}^{v} \sqrt[n]{v / K} d v$

a)

$$
W=K^{-1 / n} \frac{n}{n+1} v^{(n+1) / n}
$$

$\ln W=-\frac{1}{n} \ln K+\ln \frac{n}{n+1}+\frac{n+1}{n} \ln v$

b) $\quad \mathscr{\varphi}_{W} \equiv \frac{1}{W}=K^{1 / n} \frac{n+1}{n} v^{-(n+1) / n}$

$$
\ln \varphi_{W} \equiv \ln \frac{1}{W}=\frac{1}{n} \ln K-\ln \frac{n}{n+1}-\frac{n+1}{n} \ln v
$$

a) $\quad W_{\mathrm{s}}=K^{-1 / n} \frac{n}{n+1}$

$\ln W_{\mathrm{s}}=-\frac{1}{n} \ln K+\ln \frac{n}{n+1}$

b) $\frac{1}{W_{\mathrm{s}}}=K^{1 / n} \frac{n+1}{n}$

$\ln \varphi_{W} \equiv \ln \frac{1}{W_{\mathrm{s}}}=\frac{1}{n} \ln K-\ln \frac{n}{n+1}$

a) $\quad W=\frac{1}{A} e^{K_{W} / T}$

b) $1 / W=A e^{-K_{W} / T}$

Verhältnisse bei nicht-Newtonschen Körpern, von denen in dieser Abhandlung die Rede ist, komplizierter, da bei ihnen die Viskosität eine Funktion der Schubspannung ist.

Die Fließkurven nicht-Newtonscher Systeme können prinzipiell durch die Gln. (3) und (4) der Formelübersicht beschrieben werden. $K$ und $n$ sind Konstanten; $n$ ist größer als 1. Für $n=1$ erfolgt Übergang in einen Newtonschen Körper; 
es gelten dann die Beziehungen (1) und (2). Mit steigender Temperatur nimmt $n$ ab, so daß man annehmen darf, daß es für jeden nicht-Newtonschen Körper eine Temperatur geben wird, bei der er in ein Newtonsches System übergeht. Über die Beschaffenheit der Funktion $n=f(T)$ dürfte wenig bekannt sein.

Es wird angenommen, daß die Fließkurven nicht-Newtonscher Körper bei höheren Schubspannungen und entsprechend hohen Fließgeschwindigkeiten in Geraden übergehen. Der Übergang ist im Gegensatz zu den nämlichen Verhältnissen bei strukturviskosen Lösungen ${ }^{5}$ experimentell nicht leicht feststellbar. Infolgedessen ist die Schubspannung, bei der nicht-Newtonsches in Newtonsches Fließen übergeht, kaum als ein Kriterium zu verwenden. Man ist bei der Auswertung der hier diskutierten Fließkurven vorläufig auf den Bereich mittlerer Fließgeschwindigkeiten angewiesen, wo die Kurven ihre größte Durchbiegung zeigen. Wie man auch hier zu brauchbaren Ergebnissen gelangt, soll nunmehr gezeigt werden.

\section{III.}

Die in diesem und den folgenden Abschnitten niedergelegten Resultate und Erfahrungen beruhen hauptsächlich auf Untersuchungen an einer polymerhomologen Reihe von Polyvinylacetaten.*

Abb. 2 zeigt ein Fließkurvendiagramm für ein Polyvinylacetat mittleren Polymerisationsgrades. Die Kurven steigen sämtlich überproportional mit der Schubspannung an, mit zunehmender Temperatur verschieben sie sich nach Bereichen niederer Schubspannungen.

Die Fläche, die eine jede Fließkurve mit der Ordinate bis zu einer konventionellen Fließgeschwindigkeit einschließt, hat die Dimension einer Leistung pro $\mathrm{cm}^{2}$; sie wurde als Verformungsleistung $W$ bezeichnet. Für ihren reziproken Wert $1 / W$ schlagen wir den Begriff „Verformbarkeit“ $\varphi_{W}$ vor. Es gelten die Definitionsgleichungen $\left(5_{1}\right)$.

Alle in unseren früheren Abhandlungen über die Rheologie organischer Gläser mitgeteilten und mit der Verformungsleistung bzw. der Verformbarkeit zusammenhängenden Größen wurden empirisch ermittelt und in willkürlichen Einheiten angegeben. Inzwischen haben wir nun eine Be-

5 W. Philipp off, Die Viskosität der Kolloide, Dresden u. Leipzig 1942, S. $113 \mathrm{ff}$. rechnung der Verformungsleistung vorgenommen. Dazu ist nur die Kenntnis zweier Konstanten, die sich aus den Fließkurven ableiten lassen, erforderlich.

Der für nicht-Newtonsche Systeme vorgeschlagene Ansatz $\left(3_{1}\right)$ kann leicht auf seine Gültigkeit geprüft werden. Bei doppeltlogarithmischer Auftragung müssen die Fließkurven der Abb. 2 nach

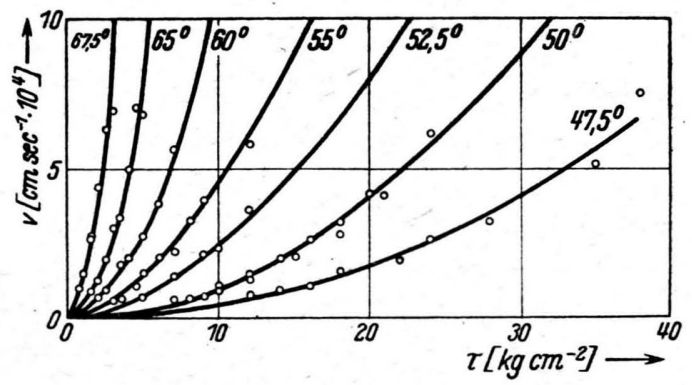

Abb. 2. Fließkurvendiagramm eines Polyvinylacetats mittleren Durchschnittspolymerisationsgrades.

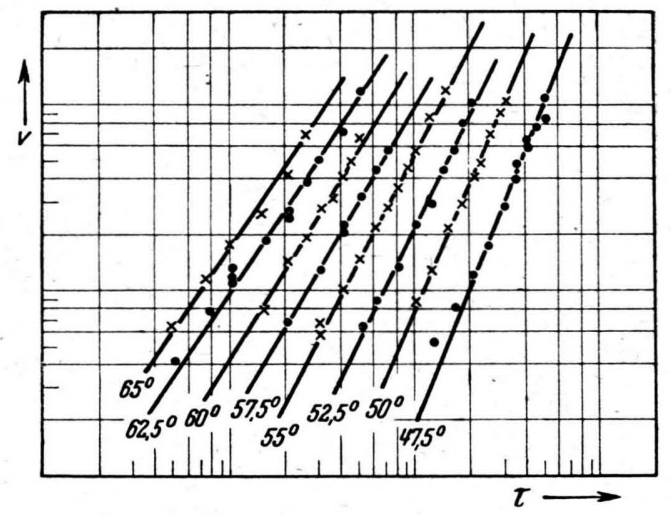

Abb. 3. Fließkurven eines Polymerisats in doppeltlogarithmischer Auftragung.

$\left(3_{2}\right)$ in Gerade übergehen. Abb. 3 gibt eine ausgezeichnete Bestätigung dieser Forderung. Im übrigen erkennt man, daß die Neigung der Kurven mit wachsender Temperatur abnimmt (Abhängigkeit des Exponenten $n$ in (3) von der Temperatur). Der Ordinatenabschnitt der doppeltlogarithmischen Fließkurven ist gleich dem Logarithmus der Konstanten $K$ in (3), nämlich die Fließgeschwindigkeit für die Einheit der Schubspannung. Sie läßt sich nach $\left(3_{2}\right)$ leicht berechnen, wenn $n$ bekannt

* Es sind das die Produkte B 55, B 17, B 60, B 100, $\mathrm{B} 1000$, U 4, U 10, U 20, U 30 , U 50, U 100 und U 1000 der Firma Dr. Alexander Wacker, München. Für die Ǔberlassung der Präparate sprechen wir der genannten Firma verbindlichsten Dank aus. 
ist. $n$ selber ist der tang des Neigungswinkels der Kurven in Abb. 3.

Es ist bekannt, daß (3) für manche nicht-Newtonsche Körper gültig ist ${ }^{6}$. Wir untersuchten eine Reihe stofflich verschiedenster nicht-Newtonscher Systeme, wie Polymerisate, geblasene Bitumina u. ä.m., und fanden immer, daß man bei der Beschreibung der experimentell ermittelten Fließkurven mit nur eínem Exponenten auskommt. Wir betonen das, weil wir den Ansatz (3) zur Berechnung der Verformungsleistung heranziehen.

Substituiert man in $\left(5_{1}\right) \tau$ mittels $\left(3_{1}\right)$, so erhält man für die Verformungsleistung bzw. die

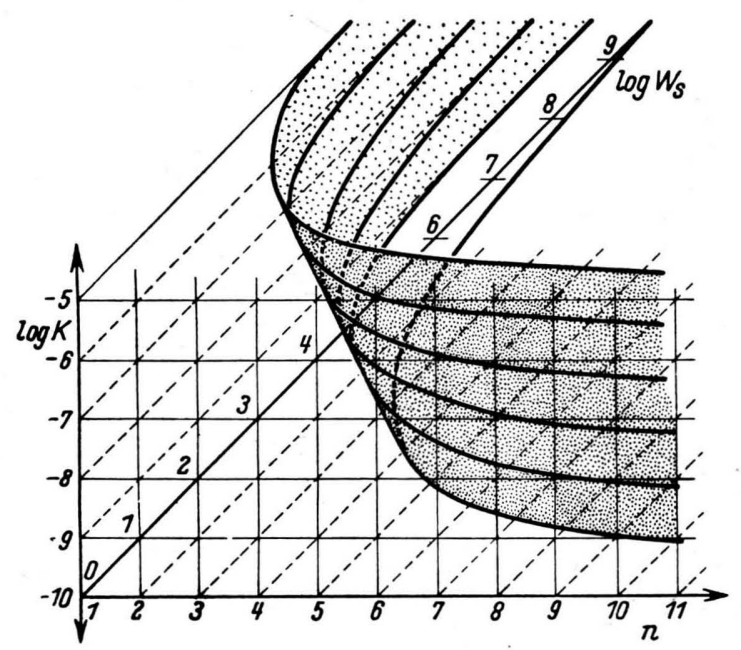

Abb. 4. Zusammenhang zwischen der Konstanten $K$ und dem Exponenten $n$ in Gl. (3) und der nach $\left(5_{3} \mathrm{a}\right)$ berechneten spezif. Verformungsleistung $W_{\mathrm{s}}$.

Verformbarkeit die Ausdrücke $\left(5_{2} a\right)$ und $\left(5_{2} b\right)$. In ihnen kommen neben $v$ nur die Konstanten $K$ und $n$ vor. Zur Berechnung der Verformungsleistung bedarf es also nur ihrer Ermittlung aus den experimentellen Fließkurven.

Um vergleichbare Verhältnisse $\mathrm{zu}$ schaffen, liegt es nahe, in $\left(5_{2} \mathrm{a}\right)$ und $\left(5_{2} \mathrm{~b}\right) v=1 \mathrm{zu}$ setzen. Man berechnet auf diese Weise die spezif. Verformungsleistung $W_{\mathrm{s}}$ bzw. die spezif. Verformbarkeit $\varphi_{\boldsymbol{w}_{\mathbf{s}}}$, für' die die Beziehungen $\left(5_{3} \mathrm{a}\right)$ und $\left(5_{3} \mathrm{~b}\right)$ gelten.

Eine anschauliche Vorstellung von den Zusammenhängen erhält man, wenn die Abhängigkeit des Logarithmus der spezif. Verformungsleistung vom Exponenten $n$ für verschiedene praktisch auftretende Werte des $\log K$ in ein Raumkoordi-

${ }^{6}$ R. Houw ink ${ }^{3}$ [1934]; T. A l fre ${ }^{1}$, S. 320 . natensystem eingetragen wird (Abb. 4). Wenn man Fließkurven, bei denen $n<1$ ist, von den Betrachtungen ausschließt, haben alle Kurven für $n=1$ ein Ende.

\section{IV.}

Der besondere Wert der entwickelten Auswertung der Fließkurvendiagramme nicht-Newtonscher Körper beruht auf der Möglichkeit, die Verformungsleistung bzw. die Verformbarkeit als Funktion der Temperatur darstellen zu können. Trägt man $\ln W$ oder $\ln 1 / W$ gegen $1 / T$ auf, so erhält man Geraden. Abb. 5 zeigt die Temperaturfunktionen einiger Polyvinylacetate. In dem hier betrachteten Temperaturbereich ist der lineare

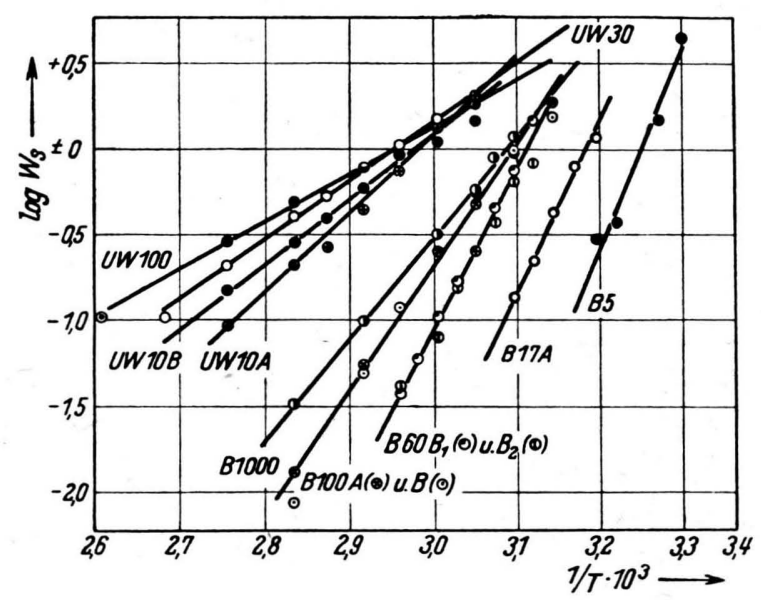

Abb.5. Die Temperaturfunktionen der Verformungsleistung einiger Polyvinylacetate unterschiedlichen Durchschnittspolymerisationsgrades.

Charakter der Kurven unverkennbar. Ob er erhalten bleibt, wenn größere Temperaturbereiche in Betracht gezogen werden, müßte freilich besonders untersucht werden. Vielleicht liegen die Verhältnisse ähnlich wie bei den ViskositätsTemperaturfunktionen von Schmelzen ${ }^{7}$. Vorerst gelten die Beziehungen (6a) und (6b). Die Richtungskonstante der Temperaturfunktion der Verformungsleistung $K_{W}$ entspricht einer Aktivierungsenergie und vermittelt direkt einen Einblick in die energetischen Verhältnisse beim Fließprozeß eines nicht-Newtonschen Körpers.

Die Temperaturabhängigkeit der Verformungsleistung hängt eng mit der Temperaturfunktion der Konstanten $K$ und $n$ in (3) zusammen. Bei Kenntnis der letzteren müßte es möglich sein, die

' E. J en c k e l, Z.physik. Chem., Abt. A 184, 309 [1939]. 


\begin{tabular}{|c|c|c|c|c|c|c|c|}
\hline \multirow{11}{*}{ 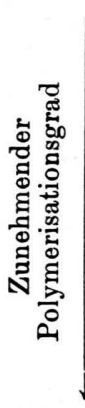 } & \multirow{2}{*}{ Polymerisat } & \multicolumn{4}{|c|}{$K_{W} \cdot 10^{-3}$} & \\
\hline & & $v=1$ & $v=10^{-1}$ & $v=10^{-z}$ & $v=10^{-3}$ & $v=10^{-4}$ & $v=10^{-s}$ \\
\hline & В 5 & 16,0 & 17,6 & 20,6 & 22,8 & 24,9 & 28,9 \\
\hline & B 17 & 12,7 & 14,8 & 17,2 & 19,6 & 22,0 & 25,0 \\
\hline & B 60 & 13,0 & 14,6 & 16,1 & 18,0 & 20,0 & 23,0 \\
\hline & B 100 & 10,0 & 11,6 & 13,5 & 15,1 & 16,6 & 19,0 \\
\hline & B 1000 & 7,0 & 8,7 & 9,6 & 12,1 & 13,7 & 16,3 \\
\hline & U 10 & 4,5 & 5,1 & 5,8 & 8,0 & 8,9 & 10,2 \\
\hline & U 30 & 3,2 & 4,3 & 5,5 & 6,5 & 7,9 & 9,0 \\
\hline & U 50 & 2,1 & 3,0 & 4,8 & 7,1 & 8,8 & 9,0 \\
\hline & U 100 & 3,1 & 4,9 & 5,3 & 5,5 & 6,5 & 7,4 \\
\hline
\end{tabular}

Tab. 1. Die Richtungskonstanten der Temperaturfunktion der mittels $\left(5_{2} a\right)$ für verschiedene Werte von $v$ berechneten Verformungsleistung.

Beziehungen (6) abzuleiten. Die Abb. 6 und 7 zeigen diese Temperaturabhängigkeit (Ordinate: $\log K$ bzw. $n$; Abszisse: $1 / T$ ) bei den untersuchten Polyvinylacetaten sowie einem Erdölbitumen (Kurve „Bit.“ in Abb. 6). In den jeweils einge-

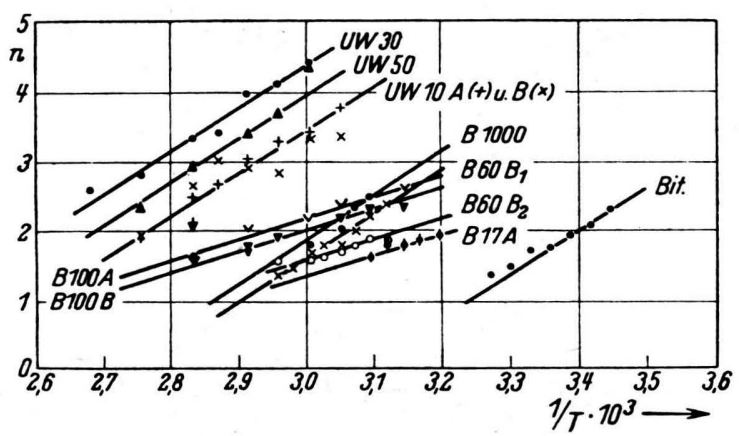

Abb. 6. Abhängigkeit' des Exponenten $n$ in (3) von der Temperatur.

stellten Temperaturbereichen ist trotz der zuweilen nicht unerheblichen Streuungen der Punkte der lineare Kurvenverlauf deutlich. An Hand des vorliegenden Versuchsmaterials kann auch in diesem Falle nicht entschieden werden, ob der Kurvenhabitus bestehen bleibt, wenn die Untersuchungen sich über größere Temperaturbereiche erstrecken. Durch Extrapolation der Kurven in Abb. $6[n=f(1 / T)]$ auf $n=1$ ergeben sich auffallend niedrige Temperaturen für einen Übergang in Newtonsches Fließen. Daraus könnte man folgern, daß die Kurven vielleicht von der Geraden abweichen, wenn sie sich $n=1$ nähern. Eine Klärung der Verhältnisse kann aber nur durch noch umfangreichere Messungen erfolgen, was freilich eine Frage der apparativen Möglichkeiten ist. Davon abgesehen, sind Polyvinylacetate hierfür

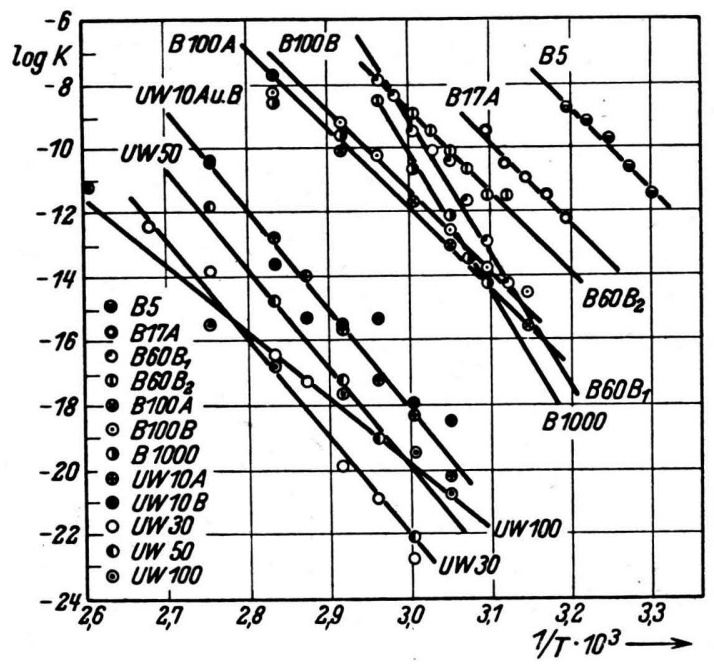

Abb. 7. Abhängigkeit der Konstanten $K$ in (3) von der Temperatur.

sicherlich kein besonders geeignetes Versuchsmaterial. Vorzügliche Modellsubstanzen für rheologische Studien sind dagegen die geblasenen Bitumina. Versuche mit ihnen könnten wesentlich zur Erforschung der Funktion $n=f(T)$ beitragen.

Auffallend ist der Unterschied in den Neigungen der Kurven in Abb. 6. Die Geraden B $100 \mathrm{~A}$ und B $100 \mathrm{~B}$ sowie B $17 \mathrm{~A}$ und B $60 \mathrm{~B}_{2}$ verlaufen viel flacher als die übrigen. Darüber hinaus besteht keine Übereinstimmung der beiden Meßreihen an Polyvinylacetat $\mathrm{B} 60$ (Kurven B $60 \mathrm{~B}_{1}$ und $\mathrm{B} 60 \mathrm{~B}_{2}$ ). Unsere bisherigen Erfahrungen mit Polymerisaten machen es wahrscheinlich, daß die Richtungskonstanten der Geraden $n=f(T)$ auch von der Vorbehandlung der Meßproben abhängen. Der Exponent $n$ ist $\mathrm{u}$. E. sicherlich auch 
durch die speziellen energetischen Verhältnisse der Feinstruktur des Polymerisats bedingt, die in quantitativer Hinsicht bei ein und derselben Temperatur je nach Vorbehandlung des in Frage stehenden Produktes gewissen Änderungen unter. worfen sein dürften. Man überzeuge sich jedoch durch Betrachtung der Abb.5, daß die beiden Meß-
V.

Die Untersuchungen wurden mit dem Konsisto. meter von $\mathrm{H}$ ö $\mathrm{p} \mathrm{p} \mathrm{le} \mathrm{r}^{8}$ ausgeführt. Wenn in einem Plastometer das Geschwindigkeitsgefälle definiert ist, errechnet man die Verformungsleistung in ab. soluten Einheiten. Beim Konsistometer von Höpp. ler ist das nicht der Fall ${ }^{8}$. Diese Feststellung be-

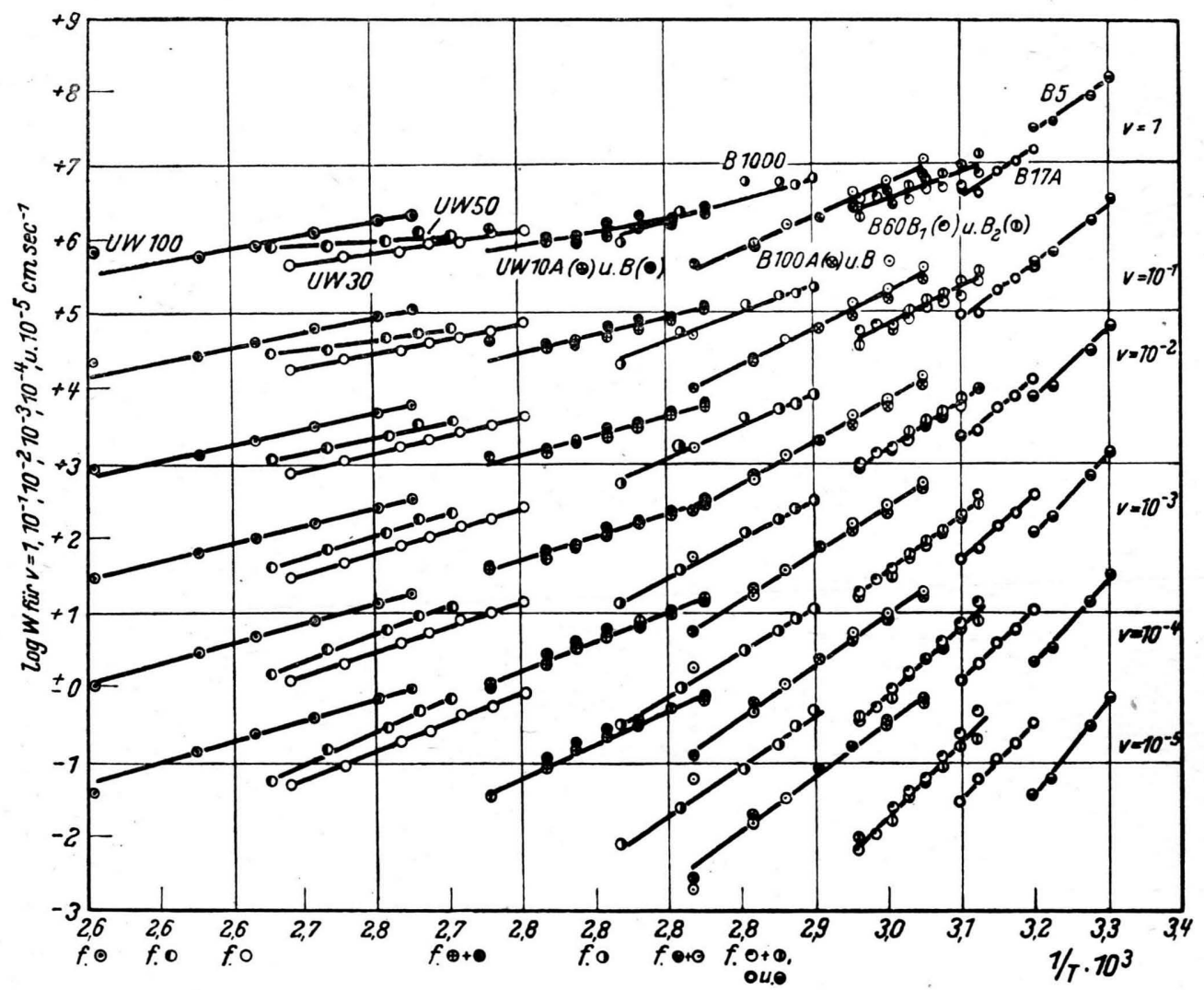

Abb. 8. Temperaturfunktionen der Verformungsleistung einiger Polyvinylacetate für verschiedene Fliefgeschwindigkeiten nach Gl. $\left(5_{2} \mathrm{a}\right)$.

reihen an B 60 zu einer einzigen Temperaturfunktion der Verformungsleistung führen.

Die Richtungskonstante $K_{W}$ der Temperaturfunktion der Verformungsleistung hängt davon $a b$, welcher Wert für die Fließgeschwindigkeit bei der Berechnung der Verformungsleistung nach $\left(5_{2} \mathrm{a}\right)$ eingesetzt wurde. $K_{W}$ nimmt mit $v$ ab. Tab. 1 enthält $K_{\dot{W}} \cdot 10^{-3}$ der einzelnen Polyvinylacetate bei verschiedenen Fließgeschwindigkeiten.

Die Abb. 8 veranschaulicht die Verhältnisse. Bei Betrachtung der Kurven beachte man die Eintragungen auf der Abszisse!

${ }^{8}$ F. Höppler, Kautschuk 17, 17 [1941]; Kunst. stoffe 31, 404 [1941]; W. Philip p of f 5 , S. 91. deutet indessen keine Einschränkung unserer Methode, Fließkurven nicht-Newtonscher Systeme auszuwerten.

Die Voraussetzung für exakte und reproduzierbare Ergebnisse ist die Einstellung des stationären Fließens. Die Deformationsgeschwindigkeit muß über ausgedehnte Deformationswege und Deformationszeiten konstant bleiben. Diese Forderung wird leider nicht von allen Polymerisaten erfüllt, z. B. nicht von plastifizierten Polyvinylchloriden, höhermolekularen Polyisobutylenen und ähn liches mehr. Aus diesem Grunde ist die rheologische Methode bei der Erforschung stofflicher Zusammenhänge in der Chemie und Physik hoch- 
polymerer Verbindungen nicht unbeschränkt anwendbar.

Zur Messung der Fließgeschwindigkeiten ist es ferner erforderlich, daß die jeweils untersuchten Polymerisatproben sich hinsichtlich ihrer übermolekularen Struktur im ,Gleichgewicht“ befinden. Man erreicht dies, indem man das Material längere Zeit „tempert", wobei die Temperatur, bei der dieses geschieht, für jeden Stoff in Erfahrung gebracht werden muß. Alsdann wird langsam auf die jeweilige Meßtemperatur abgekühlt, ein Prozeß, der u. U. in mehreren Etappen vorgenommen werden muß, wenn größere Temperaturintervalle durchschritten werden sollen. Nur durch derart vorsichtig vorgenommene Vorbehandlungen der Meßproben kann man die inneren Spannungen soweit beseitigen, daß sie das Ergebnis nicht mehr wesentlich beeinflussen. Die Temperaturfunktionen der Verformungsleistung der Polyvinylacetate B 60 und B 100 in Abb. 5 bestätigen das; auf den Kurven fallen jeweils zwei voneinander unabhängige Meßreihen zusammen.

\section{VI.}

Ein sehr wesentliches Ergebnis unserer rheologischen Untersuchungen ist die Aufdeckung eines Zusammenhanges zwischen der Richtungskonstanten der Temperaturfunktion der Verformungsleistung und dem Polymerisationsgrad. Mit wachsendem mittleren Polymerisationsgrad nimmt die Richtungskonstante der Temperaturfunktion der Verformungsleistung ab. Sobald die Durchschnittspolymerisationsgrade bzw. -molgewichte unserer Polyvinylacetate bekannt sind, werden wir eingehender darüber berichten. Es läßt sich aber schon jetzt übersehen, daß sowohl die Voluminosität nach Bredée und de B ooys, als auch die Grundviskosität $[\eta]$, berechnet mittels der Viskositätsformeln von Philipp off und
Hess bzw. G.V.Schulz und Fr.Blaschke, eine funktionelle Abhängigkeit von der Richtungskonstanten der Temperaturfunktion der Verformungsleistung zeigen. Es steht außer Zweifel, daß die im Beispiel der Polyvinylacetate gefundene Abhängigkeit zwischen Fließvermögen und Polymerisationsgrad auch bei anderen Polymerisaten vorhanden ist. Arbeiten darüber wurden in Angriff genommen.

Aufschlußreich sind auch Untersuchungen an Mischungen von Polymerisaten mit Weichmachungsmitteln. Zwei Fälle wurden bislang beobachtet:

1. Durch den Zusatz der Weichmacher wird die Verformungsleistung herabgesetzt. Gleichmolare Zusätze homologer Weichmachungsmittel bewirken eine um so größere Depression der Verformungsleistung, je höher ihr Molgewicht ist. Die Richtungskonstante der Temperaturfunktion der Verformungsleistung ändert sich praktisch nicht. Plastifizierte Polyvinylacetate sind hierfür ein Beispiel.

2. Neben einer besonders großen Depression der Verformungsleistung wird auch eine deutliche Änderung der Richtungskonstanten der Temperaturfunktion beobachtet. Es wird also auch der intermolekulare Zusammenhaltsmechanismus ein anderer. Die Änderung der Richtungskonstanten erfolgt bis zu einem „kritischen“ Zusatz. Größere Konzentrationen an Weichmachern ändern die $K_{W^{-}}$ Werte nicht mehr. Die molare kritische Konzentration ist um so kleiner, je höher das Molgewicht des Weichmachers ist. Ein Beispiel hierfür bieten Mischungen von Polystyrol mit Phthalsäureestern.

${ }_{9}^{9}$ H. L. B r e dé e u. J. de B o o y s, Kolloid-Z. 79, 31, 43 [1937], ebenda 99, 171 [1942]; G. V. Schulz u. Fr. Blaschke, J. prakt. Chem. 158, 130 [1941]; K. Hess u. W. Philip off, Ber. dtsch. chem. Ges. 70, 639 [1937]. 